Tema: Solidificação / Lingotamento

\title{
MODELAGEM FÍSICA DE REMOÇÃO DE INCLUSÕES EM DISTRIBUIDOR DE LINGOTAMENTO CONTÍNUO DE TARUGOS*
}

\section{Resumo}

\author{
Felipe das Dôres Machado ${ }^{1}$ \\ Magda Beatriz Galant François ${ }^{2}$ \\ Antônio Cezar Faria Vilela ${ }^{3}$
}

No processo de fabricação de aço, o distribuidor possui grande importância na questão limpeza inclusionária, principalmente, quando se trata da produção de aços limpos (clean steel). As inclusões podem afetar negativamente as propriedades mecânicas e metalúrgicas do aço. Estudos realizados em modelos físicos e/ou matemáticos vêm sendo aplicados com intuito de propor medidas que melhorem a capacidade do distribuidor na remoção de inclusões presentes no aço. Este trabalho tem como objetivo principal qualificar o escoamento de um distribuidor e quantificar a remoção de inclusões do mesmo. Um modelo físico de um distribuidor de lingotamento contínuo de tarugos construído em acrílico e que respeita a similaridade de Froude e Reynolds é utilizado para os ensaios. A quantificação das inclusões é realizada por meio de um ensaio que envolve um sistema de injeção de partículas de polietileno na entrada no distribuidor e outro sistema de coleta dessas partículas na saída do distribuidor no qual as amostras coletadas, posteriormente, são secadas e pesadas. Com os resultados obtidos pretende-se compreender o efeito dos parâmetros propostos sobre a remoção de inclusões e permitir a utilização de uma ferramenta capaz de avaliar a qualidade de aços no que se refere à passagem de inclusões na saída do distribuidor.

Palavras chaves: Distribuidor; Remoção de inclusões; Modelagem física.

\section{PHYSICAL MODEL OF INCLUSION REMOVAL IN CONTINUOUS CASTING TUNDISH OF STEEL BILLETS}

\section{Abstract}

In the steelmaking process, the tundish has a great importance in the inclusion removal. The inclusions can afect negatively metallurgical and mechanical properties of steel. Studies that use physical and/or mathematical models have been applied with the objective to propose methods to improve the capacity of the tundish in remove inclusions found in the steel. This work have as main objective qualify the fluid flow and quantify the inclusion removal in the tundish. A physical model of a tundish of continuous billet casting machine is used in this work and it has built in acrylic respecting the Froude and Reynolds similarities. The quantification of the inclusions is made by a expirement that involves a system of injection of polyethylene particles in the inside of the tundish and another that collect these particles in the outside of the tundish. After collected the inclusions, it is dried and weigh. Achieved the results it is supposed to understand the effect of parameters proposed about inclusion removal and allow the utilization of a tool capable to estimate the quality of steel when the subject is inclusion removal.

Keywords: Tundish; Inclusion removal; Physical Modelling.

1 Eng. Químico, Mestrando, LaSid, PPGE3M ,EE, UFRGS, Canoas, Rio Grande do Sul, Brasil.

2 Eng. Químico, Dr. INPL, Pesquisadora LaSid, UFRGS, Prof. ULBRA, Porto Alegre, Rio Grande do Sul, Brasil.

3 Eng. Metalúrgico, Prof. Dr.-Ing., LaSid, DEMET, PPGE3M, EE, UFRGS, Porto Alegre, Rio Grande do Sul, Brasil.

\footnotetext{
* Contribuição técnica ao 450 Seminário de Aciaria - Internacional, 25 a 28 de maio de 2014,
} 


\section{INTRODUÇAO}

O aumento da necessidade, por parte do setor industrial, de aços com melhores características mecânico-metalúrgicas tem requerido cada vez mais um controle rígido quanto à quantidade, tamanho e morfologia de inclusões. Os padrões necessários geram consequências diretas nos processos de produção de aços. Entre elas, estão as modificações no escoamento interno do distribuidor que, como mostram alguns trabalhos, possuem forte influência nas melhora das funções do distribuidor, inclusive na flotação das inclusões [1-8].

O distribuidor tem função preponderante no processo de fabricação de aços via lingotamento contínuo. Ele é o responsável pela alimentação contínua de aço líquido para os moldes e atua como reservatório intermediário (pulmão) durante as trocas de panela. Além disso, o distribuidor é o último equipamento com volume e tempo de residência suficiente para que as inclusões não metálicas possam flotar e serem retiradas do aço por meio da absorção pela escória.

É importante ressaltar que se tratando de aços limpos, a limpeza inclusionária no distribuidor pode ser afetada tanto positivamente com as corretas escolhas dos parâmetros operacionais, quanto negativamente como, por exemplo, uma má escolha de mobiliário interno do distribuidor que possa acarretar um escoamento inadequado capaz de contaminar o aço tratado pós-metalurgia da panela e diminuir sua qualidade final.

A presença das inclusões no aço é inevitável e a sua formação é parte do processo de fabricação. Portanto, quando a quantificação das inclusões é desejada, deve-se compreender o comportamento das inclusões quanto à origem, composição, remoção, etc. A formação das inclusões envolve diversos processos, entre eles: aqueles ligados a reoxidação do metal líquido por meio da exposição ao meio oxidante (ar atmosférico), o processo de desoxidação (adição de alumínio e outros), o fenômeno de arraste de escória da panela, a emulsificação de escória no distribuidor e vários outros que podem ser encontrados na literatura [9].

Um dos caminhos que se pode seguir para o desenvolvimento de uma técnica de remoção de inclusões, são os estudos sobre o distribuidor via modelagem física com a construção do distribuidor em acrílico, em escala real (1:1), ou reduzida e que utilizam a água como fluido simulador do aço líquido. Esses ensaios respeitam critérios de modelagem física (similaridade), como por exemplo: as similaridades de Reynolds e Froude.

A modelagem física traz uma série de ferramentas que buscam simular fenômenos metalúrgicos presentes no processo de fabricação do aço. Entre os fenômenos, a remoção de inclusões está bastante presente com o papel importante de minimizar os percentuais de inclusões contidas no aço lingotado. Para isso estão sendo realizadas pesquisas em laboratórios que forneçam uma representação realística do protótipo permitindo testar novos modificadores de fluxo, diferentes geometrias, barreiras, entre outros artifícios que buscam favorecer um melhor escoamento do aço dentro do distribuidor e, consequentemente, melhorias no tempo de interação inclusão/escória [1,2,4-8].

O objetivo deste trabalho é estudar os efeitos principais e de interação dos fatores de velocidade de lingotamento, presença de tubo longo e presença de barreiras em um modelo físico do distribuidor de lingotamento contínuo de tarugos por meio da qualificação do escoamento no ensaio de linhas de fluxo e quantificação das inclusões passantes pelo veio no ensaio de remoção de inclusões.

* Contribuição técnica ao 45 Seminário de Aciaria - Internacional, 25 a 28 de maio de 2014, Porto Alegre, RS, Brasil. 


\section{MODELAGEM FÍSICA}

Estudos realizados diretamente no distribuidor de uma usina são dificultados pelo fato de que o aço apresenta opacidade, altas temperaturas de operação e processamento em equipamentos construídos com materiais que impossibilitam tecnologicamente a visualização interna do escoamento do aço. Tendo em vista que a viscosidade cinemática da água a $20^{\circ} \mathrm{C}\left(\mathrm{v}=10^{-6} \mathrm{~m}^{2} / \mathrm{s}\right)$ [10] é similar a do aço a $1600^{\circ} \mathrm{C}\left(\mathrm{v}=0,913 \times 10^{-6} \mathrm{~m}^{2} / \mathrm{s}\right)$ [10], modelos físicos que empregam água são boas ferramentas para simular o escoamento em sistemas siderúrgicos.

Para a aplicação da modelagem física é necessário que se estabeleçam critérios de similaridade (geométrica, cinemática, dinâmica, térmica e química) e das respectivas forças governantes do sistema que garantam a simulação dos fenômenos da indústria em laboratório. Para os leitores interessados recomenda-se a leitura dos trabalhos apresentados por Szekely [11,12], Mazumdar [13] e Sahai [9].

O escoamento interno do distribuidor é importante no estudo da remoção de inclusões, dado que, estas são majoritariamente removidas por um processo de flotação e possível captura pela escória. Os fenômenos envolvidos na flotação das inclusões foi apresentado por Sahai [9]. Na modelagem física alguns ensaios podem ser realizados para a qualificação e quantificação do escoamento de modo a entender o comportamento dos parâmetros envolvidos, como por exemplo, modificadores de escoamento, formato de distribuidor, velocidade de lingotamento e outros. Entre os ensaios envolvidos pode-se citar: ensaio de linhas de fluxo [14], ensaio de distribuição de tempo de residência [15], ensaio de emulsificação de escória [16], ensaio de PIV (Particle Image Velocimetry) [17], ensaio de remoção de inclusões [18] e outros.

No Laboratório de Siderurgia da UFRGS foram construídos três modelos de distribuidores em escala 1:1, com similaridade de Reynolds e Froude para fins de pesquisa e ensino, tanto na universidade quanto na indústria. Paralelamente, também foi construído um modelo de panela, em escala 1:3, com similaridade de Froude. Todos os modelos, independente do fator de escala empregado, têm apresentado bons resultados e em boa concordância com os equipamentos originais. Quando usados para fins educacionais, os modelos em escala 1:1 apresentam maior impacto na visualização dos fenômenos envolvidos.

\subsection{Modelo Físico do Distribuidor}

O modelo físico do distribuidor de lingotamento contínuo de tarugos utilizado neste trabalho pertence ao Laboratório de Siderurgia da UFRGS, construído em acrílico e em escala 1:1, segue a similaridade de Reynolds e Froude. Este modelo utiliza água como fluido de simulação do aço líquido, apresenta dois veios, tubo longo e barreiras. As dimensões detalhadas do distribuidor e do posicionamento das barreiras com suas respectivas medidas são apresentadas no trabalho de Daoud et al. [17]. A Figura 1 mostra uma representação esquemática do modelo empregado no estudo. Maiores detalhes do modelo físico e dos ensaios apresentados a seguir podem ser encontradas em Machado FD (2014) [19].

* Contribuição técnica ao 45 Seminário de Aciaria - Internacional, 25 a 28 de maio de 2014, Porto Alegre, RS, Brasil. 

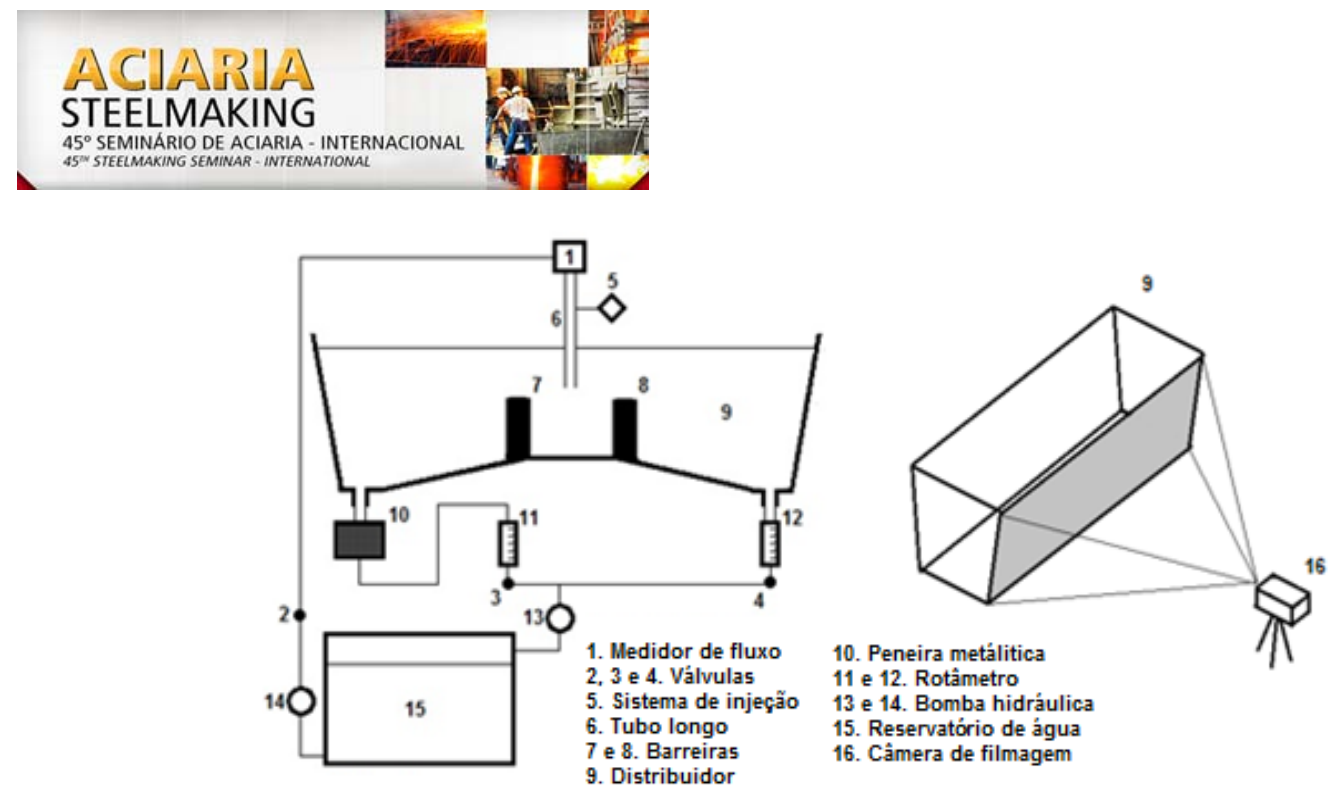

Figura 1. Representação esquemática do modelo físico de distribuidor de lingotamento contínuo e posicionamento da câmera na vista frontal.

Como pode ser visualizado na Figura 1, o modelo físico é dotado de medidores de fluxo na entrada do distribuidor e na saída de cada veio estão acoplados os rotâmetros para que seja possível controlar o regime permanente correspondente à velocidade de lingotamento que deseja simular. A água utilizada para o ensaio é oriunda de um reservatório que por meio de tubulações e bombas hidráulicas é inserida e retirada do distribuidor. O sistema de injeção é posicionado na entrada do distribuidor para utilização nos ensaios de linhas de fluxo e remoção de inclusões. Para utilização no ensaio de linhas de fluxo, é posicionada uma câmera para a captura da vista frontal do modelo do distribuidor.

A Tabela 1 exibe os parâmetros de ensaio e os intervalos de pesquisa empregados nos ensaios de linhas de fluxo e remoção de inclusões. Devido ao ensaio de linhas de fluxo ser preferencialmente qualitativo a velocidade de lingotamento utilizada nos ensaios realizados é a intermediária $(2,2 \mathrm{~m} / \mathrm{min})$. A realização dos ensaios é feita de forma aleatória tanto para os ensaios de remoção de inclusões quanto para os ensaios de linhas de fluxo.

Tabela 1. Parâmetros de processo e intervalo de pesquisa

\begin{tabular}{|llll|}
\hline Parâmetro do processo & \multicolumn{3}{l|}{ Intervalo de pesquisa } \\
\hline Velocidade de lingotamento & $1,8 \mathrm{~m} / \mathrm{min}$ & $2,2 \mathrm{~m} / \mathrm{min}$ & $2,6 \mathrm{~m} / \mathrm{min}$ \\
Presença de tubo longo no & Sem & --- & Com \\
distribuidor & Sem & --- & Com \\
Presença de barreiras & &
\end{tabular}

\subsection{Ensaio de Linhas de Fluxo}

O ensaio de linhas de fluxo é realizado com o distribuidor operando em regime permanente (taxa de acúmulo igual à zero) e para a visualização do escoamento é injetada uma solução aquosa saturada de permanganato de potássio $\left(\mathrm{KMnO}_{4}\right)$ na forma de pulso (3 segundos). A injeção ocorre após um período de estabilização do escoamento para que não haja interferências externas ao experimento.

\subsection{Ensaio de Remoção de Inclusões}

O distribuidor é mantido em regime permanente e após a estabilização do escoamento é realizada a injeção das partículas de simulação de inclusões alumina na forma de pulso (3 segundos). Para formar uma mistura homogênea capaz de ser

* Contribuição técnica ao $45^{\circ}$ Seminário de Aciaria - Internacional, 25 a 28 de maio de 2014, 
injetada no distribuidor é realizada a junção de uma amostra de polietileno previamente homogeneizada de massa igual a $30 \mathrm{~g}$ a uma solução de $80 \mathrm{ml}$ de álcool etílico absoluto, cujo volume total é igual a $150 \mathrm{ml}$. Todas as condições de ensaios (Tabela 1) são realizadas em duplicata. A massa específica do polietileno utilizado é de $960 \mathrm{~kg} / \mathrm{m}^{3}$. Por meio da Equação 1 apresentada por Sahai [11], a distribuição de diâmetro de partícula das inclusões de alumina correspondentes na usina ao utilizar micropartículas de polietileno é mostrada na Tabela 2.

$$
\frac{\mathrm{R}_{\text {inc,m }}}{\mathrm{R}_{\text {inc,p }}}=\lambda^{0,25}\left[\frac{1-\frac{\rho_{\text {inc, }}}{\rho_{\text {aço }}}}{1-\frac{\rho_{\text {inc, }}}{\rho_{\text {água }}}}\right]^{0,5}
$$

Onde, a relação entre o raio da inclusão no modelo $\left(R_{\text {inc,m }}\right)$ e o raio da inclusão na usina $\left(R_{\text {inc,p }}\right)$ considera o fator de escala $(\lambda)$ e as relações de massa específica da inclusão na usina $\left(\rho_{\text {inc,p }}\right)$ e do aço $\left(\rho_{\text {aço }}\right)$ com a inclusão no modelo $\left(\rho_{\text {inc,m }}\right)$ e a água ( $\left.\rho_{\text {água }}\right)$.

Tabela 1. Distribuição de tamanho de partícula

\begin{tabular}{|l|cccccc|}
\hline Diâmetro de partícula no modelo $(\mu \mathrm{m})$ & $<600$ & $<500$ & $<425$ & $<300$ & $<212$ & $<106$ \\
Diâmetro de partícula na usina $(\mu \mathrm{m})$ & $<170,8$ & $<142,4$ & $<121$ & $<85,4$ & $<60,4$ & $<30,2$ \\
Composição cumulativa $(\%)$ & 98 & 91 & 81 & 43 & 18 & 3 \\
\hline
\end{tabular}

Para a escolha do tempo do ensaio de remoção de inclusões são realizados ensaios de distribuição de tempo de residência para todas as configurações empregadas no modelo do distribuidor. As curvas de distribuição de tempo de residência são obtidas por meio da injeção de um traçador (ácido clorídrico) na entrada do distribuído em forma de pulso (3 segundos) e aferido a condutividade na saída do modelo do distribuidor ao longo do tempo. Na Figura 2 são apresentados os resultados da concentração adimensional em função do tempo adimensional dado pela razão do tempo de ensaio e o tempo de residência teórico $(\bar{t})$.

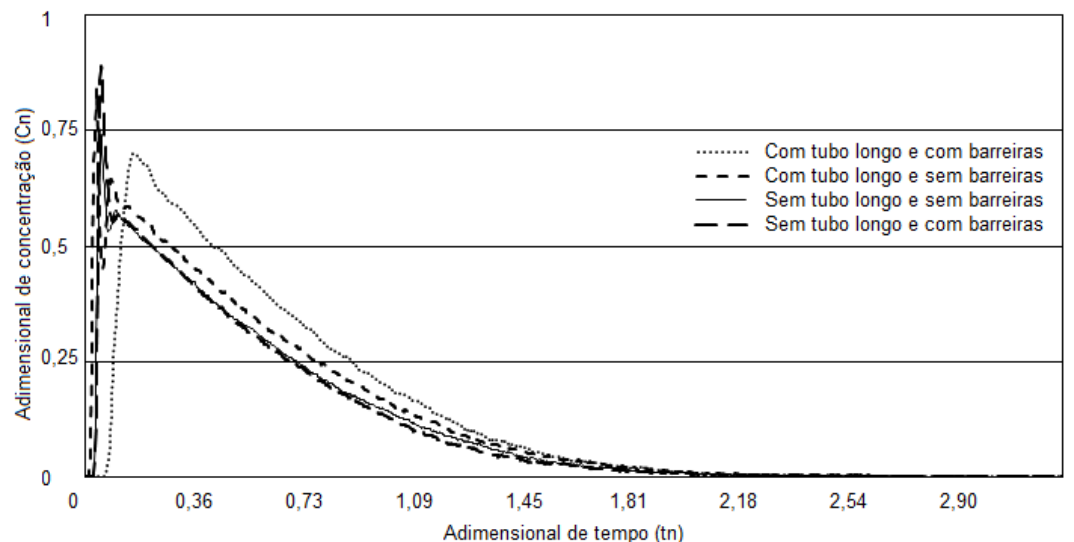

Figura 2. Curvas de distribuição de tempo de residência (DTR) no modelo do distribuídos para diferentes configurações.

Como resultado (Figura 2), mais de $97,5 \%$ do traçador utilizado no ensaio de distribuição de tempo de residência, em todos os casos empregados no modelo do distribuidor, é passante pelo veio em dois tempos de residência teóricos. Portanto, o tempo de duração do ensaio de remoção de inclusões é determinado por dois tempos de residência teóricos $(\bar{t})$, cujo cálculo é dado pela Equação 2.

* Contribuição técnica ao 450 Seminário de Aciaria - Internacional, 25 a 28 de maio de 2014, Porto Alegre, RS, Brasil. 


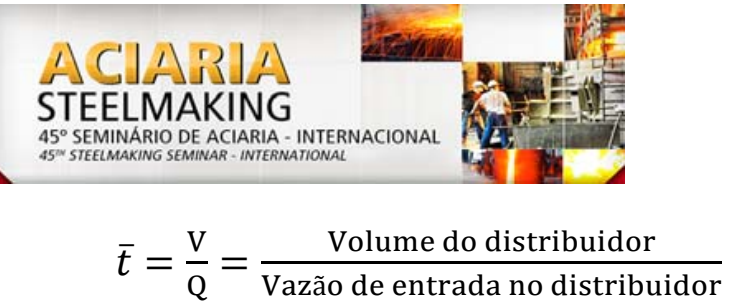

Por razões de simetria, é acoplada uma peneira metálica em somente um dos veios com o objetivo de capturar as micropartículas de polietileno no ensaio de remoção de inclusões (Figura 1). A peneira permite a passagem de partículas menores do que $37 \mu \mathrm{m}$.

O método de quantificação das inclusões via peneira tem como objetivo capturar as partículas nos veios de saída do modelo físico para posterior secagem e pesagem. O cálculo do percentual das inclusões passantes $\left(\eta_{r}\right)$ é dado pela razão entre a massa coletada no filtro $\left(m_{c}\right)$ e a massa injetada no ensaio $\left(m_{i}\right)$, conforme Equação 3.

$$
\eta_{r}(\%)=\left(\frac{m_{c}}{m_{i}}\right) \times 100
$$

\section{RESULTADOS E DISCUSSÃO}

Inicialmente são apresentados resultados de qualificação do escoamento e depois os resultados de quantificação de inclusões. Os resultados de qualificação fornecem melhoram do entendimento dos processos que envolvem a flotação das inclusões permitindo uma melhor compreensão dos resultados de flotação.

\subsection{Ensaio de linhas de fluxo}

Os resultados do ensaio de linha de fluxo são apresentados a seguir nas configurações possíveis entre a presença ou não das barreiras e tubo longo. Nos ensaios apresentados nas figuras 3 a 6, o tempo é mostrado na forma de escalas de cinza. Quanto mais claro o tom de cinza, maior é o tempo de ensaio.

\subsubsection{Distribuidor sem tubo longo e sem barreiras}

Para a configuração sem tubo longo e sem barreiras (Figura 3), a penetração do jato é pequena devido à quebra dos vetores de velocidade gerada pelo choque com a interface ar/água. Além disso, essa configuração permite ao escoamento um movimento ascendente e um maior contato do aço com a escória antes do escoamento se dirigir para os veios. No entanto, o escoamento se divide em dois: um ascendente que se espalha pela superfície e outro descendente que se direciona diretamente aos veios, caracterizado como curto-circuito.

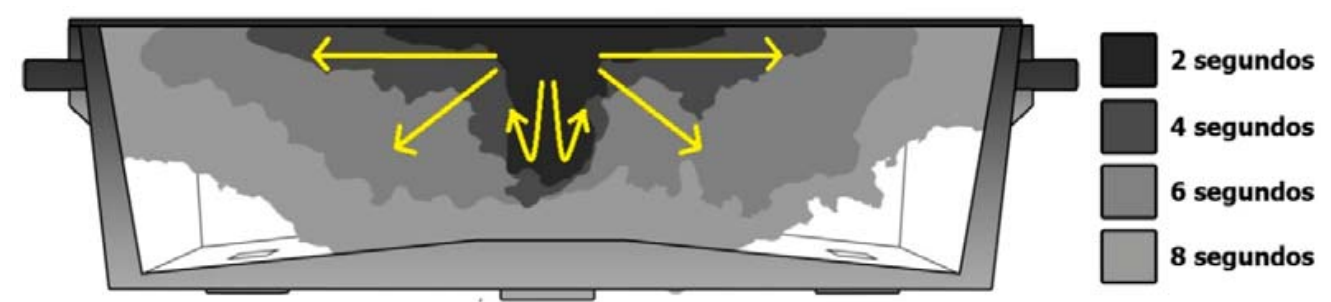

Figura 3. Ensaio de linha de fluxo para a configuração sem tubo longo e sem barreiras.

\subsubsection{Distribuidor sem tubo longo e com barreiras}

Quando são empregadas as barreiras e não é utilizado o tubo longo (Figura 4), o escoamento ocorre também de forma ascendente. Entretanto, há uma menor velocidade de escoamento na superfície do distribuidor causada pelo choque do jato com a interface ar/água e superfície das barreiras. Esse escoamento mais lento

* Contribuição técnica ao 45 Seminário de Aciaria - Internacional, 25 a 28 de maio de 2014, Porto Alegre, RS, Brasil. 


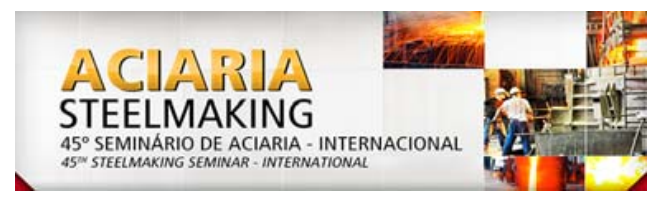

apresenta menor contato com superfície do distribuidor antes de se direcionar para os veios de saída. O escoamento novamente se divide em dois: um ascendente que se espalha pela superfície e outro descendente que se direciona diretamente aos veios caracterizando o curto-circuito.

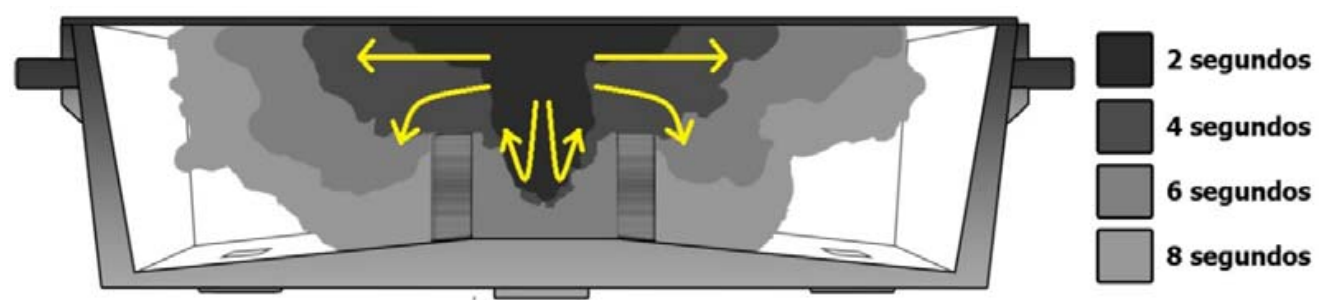

Figura 4. Ensaio de linha de fluxo para a configuração sem tubo longo e com barreiras.

\subsubsection{Distribuidor com tubo longo e sem barreiras}

Se adicionado o tubo longo e retirada as barreiras (Figura 5), o aço atinge o fundo do distribuidor mais facilmente devido à ausência da quebra dos vetores de velocidade gerada pelo choque do jato com a interface ar/água. Essa maior velocidade faz com que o escoamento descendente se dirija diretamente ao fundo do distribuidor sem passar pela superfície do distribuidor e atingindo o veio em aproximadamente 4 segundos. Este comportamento caracteriza um curto-circuito que é prejudicial à qualidade do aço. O escoamento em curto-circuito se caracteriza pelo movimento do fluido direcionado diretamente do veio à válvula, com um tempo de residência muito inferior ao tempo de residência do fluido no reator. As consequências disto estão ligadas a uma maior variação de temperatura nos veios e um maior índice de sujidade no tarugo proveniente deste veio.

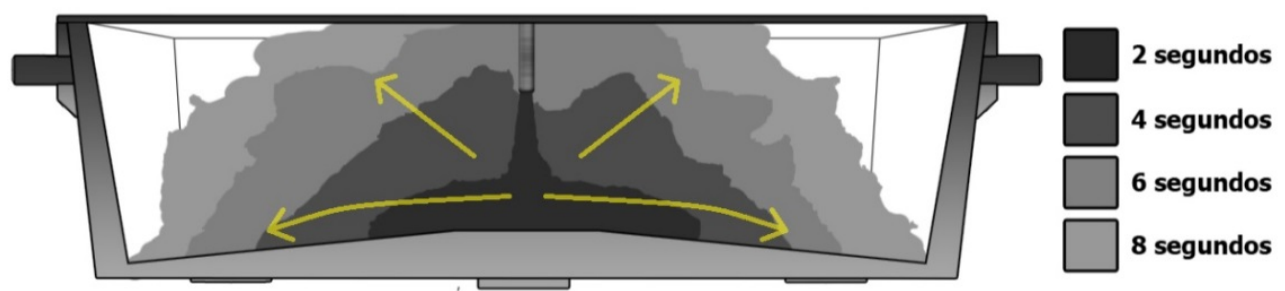

Figura 5. Ensaio de linha de fluxo para a configuração com tubo longo e sem barreiras.

\subsubsection{Distribuidor com tubo longo e com barreiras}

Quando há a presença do tubo longo e das barreiras (Figura 6) o escoamento se torna mais próximo do ideal. O escoamento é, novamente, de característica ascendente, atinge o fundo do distribuidor e espalha sobre a superfície. Após, o corante entra em contato com toda a escória e somente então se direciona para os veios. Diferentemente da primeira configuração empregada (Figura 3), a quebra dos vetores de velocidade gerada pela presença das barreiras faz com que o escoamento seja muito mais lento e favorável à flotação de inclusões. Apesar de o ensaio de linhas de fluxo ser uma ferramenta quantitativa, qualitativamente é possível perceber no maior tempo de ensaio (32 s de ensaio) que essa configuração leva para o corante atingir a mesma área que as demais configurações nas Figuras 3, 4,e 5 (8 s de ensaio).

* Contribuição técnica ao 45 Seminário de Aciaria - Internacional, 25 a 28 de maio de 2014, Porto Alegre, RS, Brasil. 

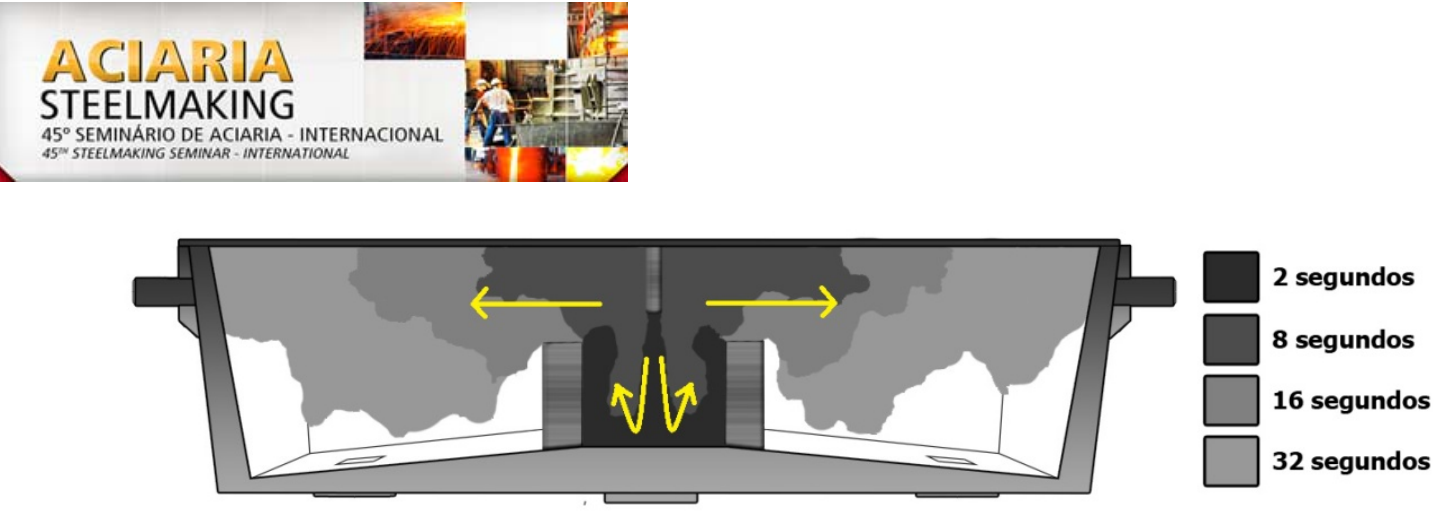

Figura 6. Ensaio de linha de fluxo para a configuração com tubo longo e com barreiras.

\subsection{Ensaio de Remoção de Inclusões}

Os resultados encontrados para os parâmetros empregados no ensaio de remoção de inclusões estão apresentados na Tabela 3. A variável resposta, percentual de inclusões passantes, deve ser entendida como a quantidade de inclusões que entram no distribuidor e atingem o veio de modo que caracterizam a passagem para o molde. Ou então, as inclusões que não são captadas pela camada de escória presente na superfície do banho.

Tabela 3. Porcentagem de inclusões passantes obtidos com a matriz experimental

\begin{tabular}{|cccccc|}
\cline { 3 - 6 } Amostra & Velocidade & Tubo & Barreiras & $\mathbf{1}$ & $\mathbf{2}$ \\
\hline 1 & $1,8 \mathrm{~m} / \mathrm{min}$ & Não & Não & $2,89 \%$ & $2,22 \%$ \\
2 & $2,2 \mathrm{~m} / \mathrm{min}$ & Não & Não & $2,35 \%$ & $3,08 \%$ \\
3 & $2,6 \mathrm{~m} / \mathrm{min}$ & Não & Não & $2,42 \%$ & $2,39 \%$ \\
4 & $1,8 \mathrm{~m} / \mathrm{min}$ & Sim & Não & $22,48 \%$ & $22,67 \%$ \\
5 & $2,2 \mathrm{~m} / \mathrm{min}$ & Sim & Não & $32,01 \%$ & $31,74 \%$ \\
6 & $2,6 \mathrm{~m} / \mathrm{min}$ & Sim & Não & $33,93 \%$ & $35,74 \%$ \\
7 & $1,8 \mathrm{~m} / \mathrm{min}$ & Não & Sim & $2,65 \%$ & $2,94 \%$ \\
8 & $2,2 \mathrm{~m} / \mathrm{min}$ & Não & Sim & $3,19 \%$ & $3,20 \%$ \\
9 & $2,6 \mathrm{~m} / \mathrm{min}$ & Não & Sim & $3,19 \%$ & $2,90 \%$ \\
10 & $1,8 \mathrm{~m} / \mathrm{min}$ & Sim & Sim & $18,43 \%$ & $18,62 \%$ \\
11 & $2,2 \mathrm{~m} / \mathrm{min}$ & Sim & Sim & $23,73 \%$ & $25,91 \%$ \\
12 & $2,6 \mathrm{~m} / \mathrm{min}$ & Sim & Sim & $28,45 \%$ & $30,61 \%$ \\
\hline
\end{tabular}

A partir dos resultados é possível isolar os efeitos principais e padronizar as escalas entre os gráficos para comparação da intensidade da influência da variável (velocidade, presença do tubo e das barreiras) sobre a variável resposta (porcentual de inclusões passantes). Na Figura 7, apresenta-se o efeito da velocidade de lingotamento sobre o porcentual de inclusões. Verifica-se que o aumento da velocidade de lingotamento permite uma maior passagem de inclusões para o veio. Este resultado é coerente, dado que o aumento da velocidade de lingotamento teoricamente aumenta o vetor de velocidade de arraste da inclusão, dificultando a sua flotação.

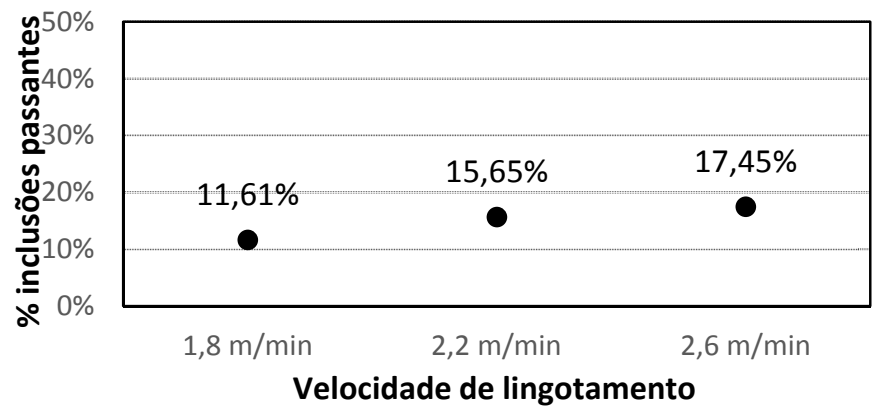

Figura 7. Porcentual de inclusões passantes em função da velocidade de lingotamento.

* Contribuição técnica ao $45^{\circ}$ Seminário de Aciaria - Internacional, 25 a 28 de maio de 2014, Porto Alegre, RS, Brasil. 


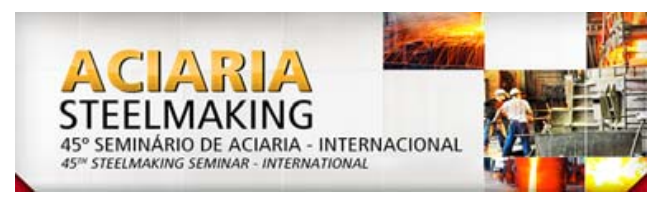

O efeito principal da presença do tubo longo (Figura 8) se mostra bastante influente sobre a quantidade de inclusões passantes pelo veio. Essa diferença nos resultados pode ser atribuída a dois fenômenos presentes no distribuidor quando ocorre a troca de jato aberto (com tubo longo) para jato fechado (sem tubo longo). O primeiro fenômeno é a quebra da velocidade do escoamento gerada pelo choque do jato com a interface ar/água. Fenômeno que pode ser visto na Figura 3 e na Figura 4, no qual o corante não atinge o fundo do distribuidor como na Figura 5 e na Figura 6 para o primeiro instante de ensaio em 2 segundos. Essa quebra de velocidade faz com que o escoamento seja mais lento e favoreça a flotação das inclusões. O segundo fenômeno, provavelmente de maior influência, remete ao arraste de bolhas gerado quando o jato atravessa a interface ar/água (Figura 9). Essas bolhas capturam as inclusões e aumentam a eficiência de remoção das inclusões.

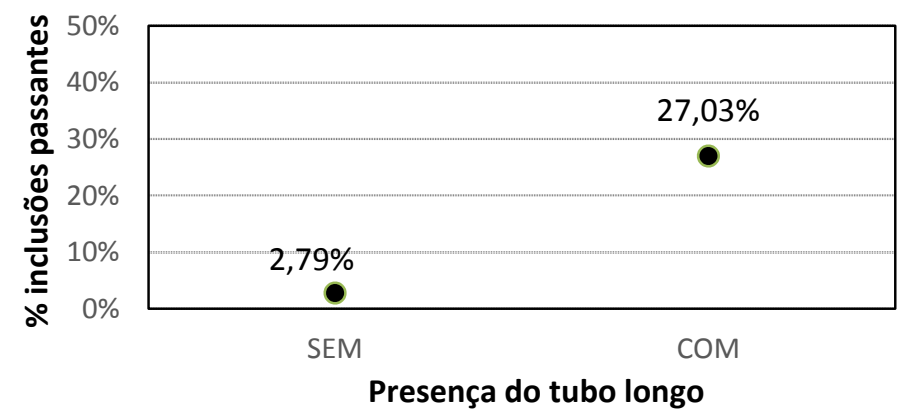

Figura 8. Porcentual de inclusões passantes em função da presença do tubo longo.

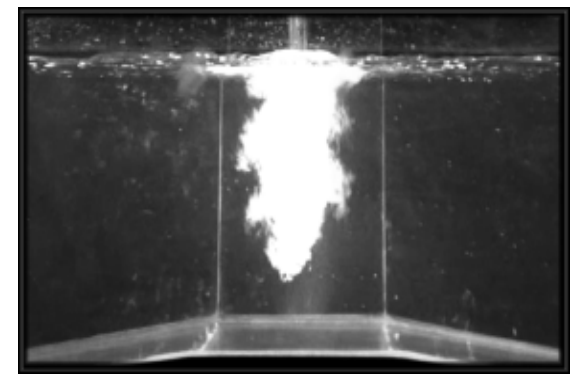

Figura 9. Arraste de bolhas para o seio do banho metálico gerado na ausência do tubo longo.

No entanto, ao abordar a presença ou não do tubo longo deve-se lembrar de que a ausência do tubo longo aumenta a formação de inclusões via reoxidação do banho metálico. Enquanto que a presença do tubo longo evita a reoxidação do aço. Logo, deve-se lembrar de que os resultados que envolvem a ausência do tubo longo não consideram a quantificação da reoxidação via exposição do aço líquido à atmosfera reoxidante. Tal quantificação poderia elevar o valor de inclusões passantes acima ou não das configurações com a presença do tubo longo.

A presença das barreiras (Figura 10) mostra um efeito negativo na passagem de inclusões para o molde e este efeito é atribuído à diminuição de velocidade do escoamento. Visto que a inclusão sofre influência de um vetor resultante para a flotação, uma diminuição no vetor de velocidade gerado pela inércia do escoamento favorece a flotação das inclusões.

* Contribuição técnica ao 45 Seminário de Aciaria - Internacional, 25 a 28 de maio de 2014, Porto Alegre, RS, Brasil. 

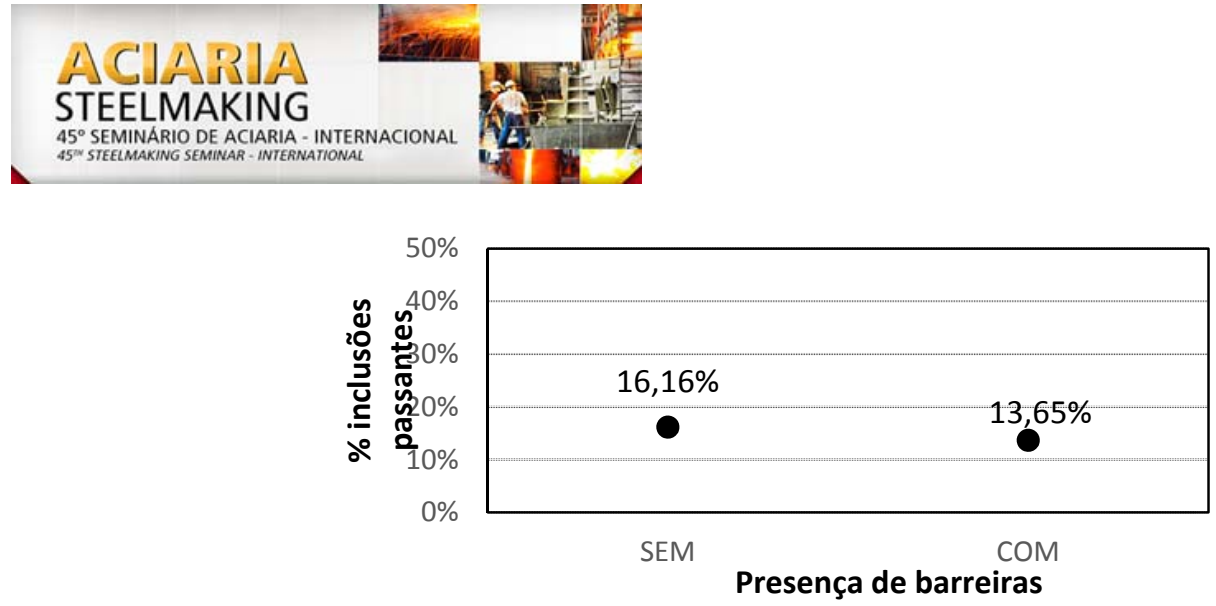

Figura 10. Porcentual de inclusões passantes em função da presença das barreiras.

Não somente os efeitos principais devem ser estudados. Os efeitos de interação são importantes para a análise das combinações entre os parâmetros. Para o caso do efeito da velocidade de lingotamento sobre a presença ou não do tubo longo (Figura 11) é possível afirmar que a presença do tubo longo é mais sensível ao aumento da velocidade de lingotamento e o valor da interação positiva e significativa é de 5,79\%. Visto que a utilização exclusiva do tubo longo apresenta um escoamento descendente com curto-circuito como mostrado nos ensaios de linhas de fluxo.

$\mathrm{Na}$ Figura 12, o efeito de interação da presença ou não das barreiras com 0 aumento da velocidade de lingotamento não se mostra significativo à passagem de inclusões não flotantes para o molde.

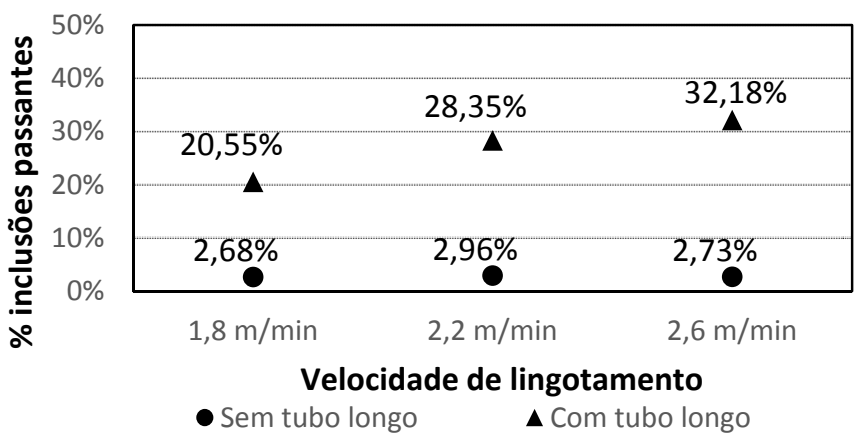

Figura 11. Porcentual de inclusões passantes em função da combinação entre a presença do tubo longo e a velocidade de lingotamento.

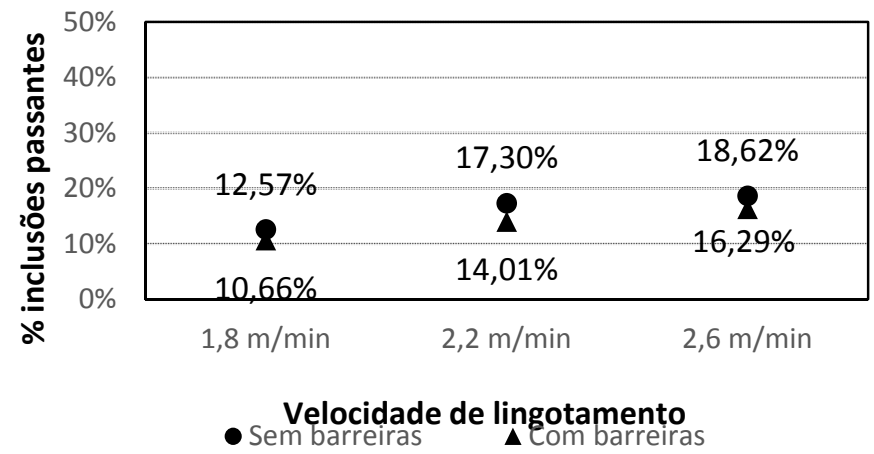

Figura 12. Porcentual de inclusões passantes em função da combinação entre a presença das barreiras e a velocidade de lingotamento.

O efeito de interação entre a presença ou não do tubo longo e a presença ou não das barreiras (Figura 13) se mostra importante na quantidade de inclusões passantes. Onde há uma interação negativa e significativa 2,96\%. A presença das barreiras evita a existência do curto-circuito gerado pela presença exclusiva do tubo longo. Assim, a quantidade de inclusões passantes pelo veio é diminuída.

* Contribuição técnica ao 45 Seminário de Aciaria - Internacional, 25 a 28 de maio de 2014, Porto Alegre, RS, Brasil. 

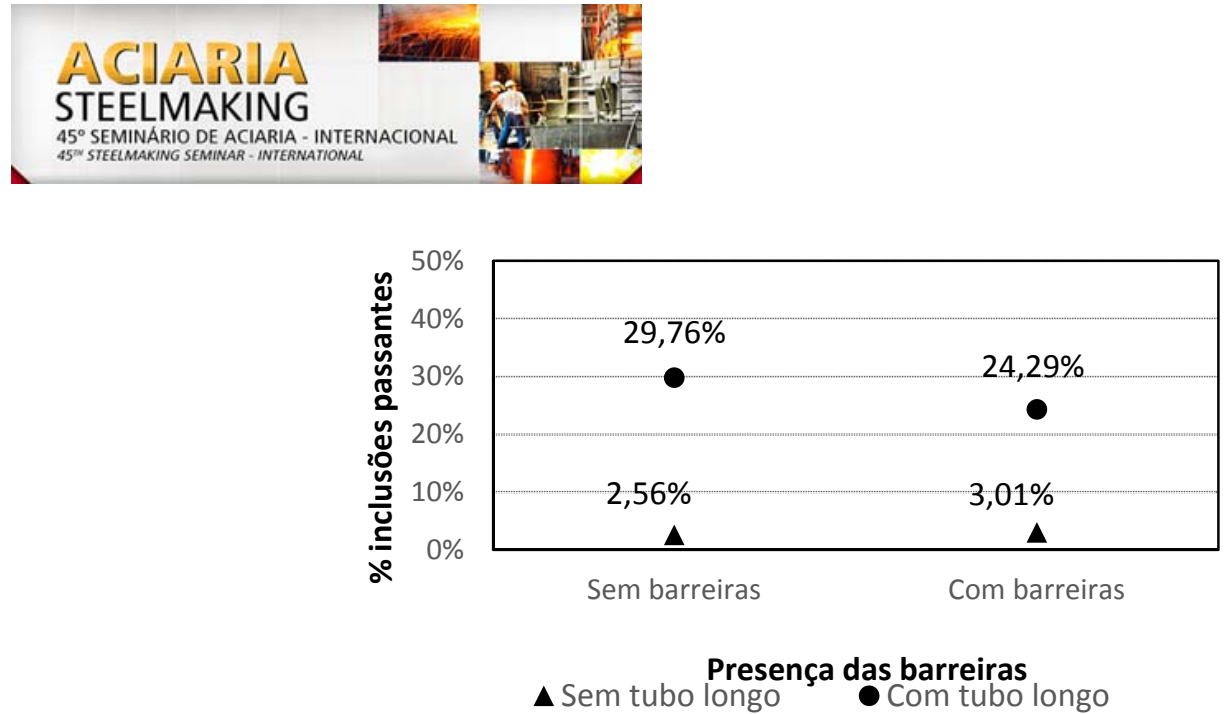

Figura 13. Porcentual de inclusões passantes em função da combinação entre a presença das barreiras e a presença do tubo longo

É importante ressaltar que esses fatores se mostraram prejudiciais ou não para a qualidade do aço, no quesito limpeza inclusionária. Entretanto, isso pode ocorrer somente para essa geometria de distribuidor ou similares. Logo, não é possível afirmar que para qualquer distribuidor com os mesmos fatores irão apresentar as mesmas consequências na variável resposta. O que se pode afirmar é que a modificação do escoamento possui efeito significativo na remoção de inclusões não metálicas no distribuidor

\section{CONCLUSÕES}

Conclui-se que o ensaio de modelagem física de remoção de inclusões em distribuidores de lingotamento contínuo de tarugos utilizando micropartículas de polietileno e água como fluido de simulação é viável do ponto de vista experimental, conforme mostrado pelos resultados obtidos em laboratório.

Com os ensaios realizados é possível afirmar que:

- O aumento da velocidade de lingotamento provoca um aumento significativo no porcentual de inclusões passantes pelo veio do modelo físico estudado (11,61\% em 1,8 m/min para $17,45 \%$ em 2,6 m/min);

- A utilização do tubo longo também apresenta efeito significativo de aumento na quantidade de inclusões no modelo físico estudado (2,79\% sem tubo longo para $27,03 \%$ com tubo longo);

- A utilização das barreiras diminui significativamente a quantidade de inclusões passantes no distribuidor utilizado (16,16\% sem barreiras para $13,65 \%$ com barreiras);

- Existe uma interação positiva e significativa entre tubo longo e velocidade de lingotamento de $5,79 \%$, onde a presença do tubo longo gera um efeito maior da velocidade de lingotamento na quantidade de inclusões do que sem a utilização da mesma;

- Há uma interação negativa e significativa entre o tubo longo e as barreiras de $2,96 \%$ que, consequentemente, gera uma diminuição na quantidade de inclusões. Por meio do ensaio de corante é visto que a presença das barreiras elimina a existência do curto-circuito gerado pela utilização exclusiva do tubo longo;

- Não há efeito de interação significativo entre a presença das barreiras e o aumento da velocidade de lingotamento.

* Contribuição técnica ao 450 Seminário de Aciaria - Internacional, 25 a 28 de maio de 2014, 


\section{NOMENCLATURA}

$\mathrm{R}_{\text {inc,m }}$ : Raio da inclusão do modelo;

$\mathrm{R}_{\text {inc,p }}$ : Raio da inclusão do protótipo;

$\lambda$ : $\quad$ Fator de escala;

$\rho_{\text {água }}$ : Massa específica da água;

$\rho_{\text {aço: }}$ assa específica do aço.

$\rho_{\text {inc,m: }}$ : Massa específica da inclusão do modelo;

$\rho_{\text {inc,p: }}$ : Massa específica da inclusão do protótipo;

$\eta_{r}: \quad$ Percentual de inclusões passantes;

$m_{c}$ : Massa coletada no filtro;

$m_{i}$ : Massa injetada no filtro;

$\bar{t}$ : $\quad$ Tempo de residência teórico;

V: $\quad$ Volume do modelo do distribuidor;

Q: Vazão empregada no ensaio.

\section{Agradecimentos}

Ao CNPq pela bolsa concedida ao aluno de mestrado do Programa de Pós Graduação de Engenharia de Minas, Metalurgia e Materiais (PPGE3M) da UFRGS Felipe das Dôres Machado. À empresa Plastécnica pela confiança e doação ao Laboratório de Siderurgia da UFRGS do material utilizado para simulação das inclusões deste trabalho. Aos bolsistas de iniciação científica do Laboratório de Siderurgia da Escola de Engenharia da UFRGS: Elisa Araujo Santos Darsie e Paula Rodrigues Caumo.

\section{REFERÊNCIAS}

1 Joo S, Guthrie RIL. Inclusion behavior and heat-transfer phenomena in steelmaking tundish operations - Part I: aqueous. Metallurgical transactions. 1993;24:755-65.

2 Cho $\mathrm{J}$, Lee $\mathrm{H}$. Cold model study on inclusion removal from liquid steel using fine gas bubbles. The Iron and Steel Institute of Japan International. 2001;41:151-7.

3 Rogler JP, Heaslip LJ, Mehrvar M. Can. Metal. Quar. 2005;44(3):357-68.

4 Jin-Gang LE. Water modeling of optimizing tundish flow field. Journal of Iron and Steel Research. 2007;14:13-19.

5 Yue Q, Zou Z-S, Hou Q-F, Chen Z. Water modeling of swirling flow tundish for steel continuous casting. Journal of Iron and Steel Research International. 2009;16:17-22.

6 Chattopadhyay K, Isac M, Guthrie RIL. Physical and mathematical modelling to study the effect of ladle shroud mis-alignment on liquid metal quality in a tundish. The Iron and Steel Institute of Japan International. 2011;51:759-68.

7 Zhang MJ, Gu HZ, Huang A, Zhu HX, Deng CJ. Physical and mathematical modeling of inclusion removal with gas bottom-blowing in continuos casting tundish. Journal of Mining and Metallurgy. 2011;47:37-44.

8 Seshadri V, Silva CA, Silva IA, Júnior ESA. A physical modelling study of inclusion removal in tundish using inert gas curtain. Tecnologia em Metalugia, Materiais e Mineração. 2012;9:22-9.

9 Sahai Y, Emi T. Tundish technology for clean steel production. World Scientific Publishing Company; 2007.

10 Mazumdar D, Guthrie ILR. The physical and mathematical modeling of continuous casting tundish systems. ISIJ Intern. 1999;39(6):524-47.

\footnotetext{
* Contribuição técnica ao 450 Seminário de Aciaria - Internacional, 25 a 28 de maio de 2014, Porto Alegre, RS, Brasil.
} 
11 Szekely J, Evans JWE, Brimacombe JK. The mathematical and physical modeling of primary metals procesing operations. Nova York: Wiley-Interscience; 1988.

12 Szekely J. Fluid flow phenomena in metals processing. Departament of materials science and engineering, Massachussetts Institute of Technology. Academic Press; 1979.

13 Mazumdar D, Evans JW. Modeling of steelmaking process. CRC Press; 2009.

14 François MBG, Daroit M, Bock ML, Mattielo MAZ, Vilela ACF. Influência do design do distribuidor nos parâmetros de escoamento. In: $36^{\circ}$ Seminário de Fusão, Refino e Solidificação dos Metais - Internacional; 2005; Vitória, Brasil. São Paulo: ABM; 2005. v.1, p.254-263.

15 François MBG, Mattielo MAZ, Bock ML, Vilela ACF. Contribuição ao entendimento das curvas de determinação de tempos característicos (DTR) e dos volumes característicos em modelos físicos de distribuidor de lingotamento contínuo. In: $15^{\circ}$ Conferencia de Aceria; 2005; San Nicolás, Argentina. San Nicolas: Instituto Argentino de Siderurgia IAS; 2005. p.327-336.

16 Mello MA, François MBG, Vilela ACF, Wollmann AM, Madias J. Effects of turbulence inhibitors on slag emulsification in the continuos casting tundish. In: Sixth International Conference on Molten Slags, Fluxes and Salts; 2000; Stockholm, Suécia: Division of Metallurgy, KTH; 2000.

17 Daoud ILA, François MBG, Vilela ACF, Van der Laan FT. Aplicação da técnica PIV em um modelo físico de distribuidor de lingotamento contínuo de aço. Proceedings of ENCIT; 29 nov.- 3 dez. 2004; Rio de Janeiro, Brasil. ABCM; 2004.

18 François MBG, Machado DFE, Vilela ACF. Remoção de inclusões em modelos físicos de distribuidores de lingotamento contínuo de aços: uma revisão bibliográfica. In: 44º Seminário de Aciaria; 2013; Araxá, Brasil. São Paulo: ABM; 2013.

19 Machado FD. Modelagem física de remoção de inclusões em distribuidor de lingotamento contínuo de tarugos. Dissertação de Mestrado. Porto Alegre: Universidade Federal do Rio Grande do Sul, Programa de Pós-Graduação em Engenharia de Minas, Metalúrgica e Materiais - PPGE3M, 2014.

* Contribuição técnica ao $45^{\circ}$ Seminário de Aciaria - Internacional, 25 a 28 de maio de 2014, 